\title{
Complications, Errors, and Surgical Ethics
}

\author{
Peter Angelos
}

Published online: 20 February 2009

(C) Société Internationale de Chirurgie 2009

\section{Introduction}

In their article titled The Ethics of Surgical Complications, Adedeji and colleagues explore the ethical dimensions of surgical complications [1]. Although there are numerous ways that one could explore the ethical aspects of such complications, these authors have used the four widely known ethical principles of respect for autonomy, beneficence, non-maleficence, and justice as the prism through which to consider surgical complications. Through this line of analysis, we see how general principles can be applied in specific situations to help guide surgeons' behaviors. The authors have done an excellent job of showing the relevance of the ethical principles for guiding action. They have also shown how focusing on a specific aspect of surgical ethics, such as complications, can help define the surgeon's proper role.

Critical to the entire manuscript is the definition of a surgical complication. The authors accept the definition of "complication" that was recently proposed in the World Journal of Surgery: "A surgical complication is any undesirable, unintended and direct result of surgery affecting the patient, which would not have occurred had the surgery gone as well as could reasonably be hoped" [2].

Using this definition of a surgical complication, the authors begin with the principle of respect for autonomy to explore the implications for surgeon behavior. It is immediately apparent that in order to respect the autonomy

P. Angelos $(\bowtie)$

Department of Surgery and MacLean Center for Clinical

Medical Ethics, The University of Chicago, 5841 S. Maryland

Avenue, MC 4052, Chicago, IL 60637, USA

e-mail: pangelos@surgery.bsd.uchicago.edu of patients, surgeons must disclose the possibility of complications when they obtain the patient's informed consent. The authors go on to emphasize that respect for autonomy also requires that complications, when they occur, must be disclosed to patients. Although the definition of a surgical complication does not carry with it any concept of error, the authors do raise this important issue when they state: "a surgeon who has made a mistake may attempt to disguise it by calling the error a surgical complication, thereby relying on the inference that this eventuality is inevitable. This is deceptive and paternalistic." I agree that to suggest that an error is merely a complication would be misleading. However, it is not clear from the article how errors and complications are actually related. We will return to this issue below.

The authors then turn to the principle of beneficence and explore the implications for surgeon behavior in applying this principle. The importance of ensuring the appropriateness of surgery can be derived from beneficence-that is, doing good for patients. Furthermore, the maintenance of a surgeon's skill and knowledge is required by the principle of beneficence. The authors also suggest that a surgeon's responsibility to address the material needs of the health care system can also be derived from the principle of beneficence. This issue may be of greater concern to surgeons working within a national health system. Finally, the important concept of surgical innovation is even addressed as being an essential aspect of furthering the good of patients.

The principle of non-maleficence, or avoiding harm, is next addressed. The authors suggest that many aspects of avoiding complications such as exercising good judgment, recognizing the limits of one's competence, infectioncontrol measures, and quality improvement programs can 
all be derived from the principle of non-maleficence. In addition, the concept of avoiding harm can be extended beyond the patient to other members of the health care team. In this fashion, non-maleficence can be used to argue for treating all members of the health care team with respect. This same line of argument is used to argue for a system of reporting deaths and complications in an environment in which a "climate of blame" is avoided. Although it would be difficult to argue that a climate of blame would be valuable, it is often difficult to remove the concept of personal responsibility from discussions of complications.

Finally, the authors look to the principle of justice and find an argument for the right of patients to have an adequate standard of surgical care. This way of looking at maintaining standards of surgical care is important because it also requires the surgeon to identify a situation in which a patient may not be getting adequate care. Such a situation may occur when a surgeon lacks the training to complete an operation. Alternatively, it may be that the surgeon's ability is impaired by substance abuse or even a decline in surgical skills with aging. In either case, the principle of justice provides a basis for surgeons to police themselves and their colleagues in order to avoid complications for patients.

Although the ethical principles utilized above are important and the behaviors that the authors suggest are derived from these principles are essential, I believe that three critical points are not adequately addressed in this article: (1) the relationship between a complication and an error; (2) the personal responsibility that surgeons feel for their patients' complications; and (3) the burden of potential complications upon individual surgeons.

\section{Relationship Between Complications and Errors}

By virtue of the definition of "complication" used, the authors seem to attempt to distinguish surgical complications from errors. This distinction is an important one to explore, because often complications occur as a result of errors. Errors can even occur without there being an associated complication. However, an error clearly suggests the possibility of culpability. For example, if in the course of performing a thyroidectomy, my patient sustains a recurrent laryngeal nerve injury, a complication has clearly occurred. The question, however, remains whether an error occurred. Whether I committed an error would be dependent on the manner in which I conducted the operation. Did I exercise due care? Did I employ the important maneuvers to avoid injuring the nerve? If so, although a complication may have occurred, an error may not have been committed.
The above statement suggests an important manner in which complications and errors are related: complications occur, whereas errors are committed. Despite the goal of avoiding a climate of blame, any discussion of errors suggests a greater sense of personal responsibility than does the discussion of a complication. One way to think about surgical errors is to think of an error as being a deviation from the intended method of doing something. In the modern approach to a systems-based method of analyzing errors, one often sees the attempt to identify sources of error in a blameless manner. This approach seeks to find the causes of error without simply blaming a particular person responsible for the error. This approach can encourage health care providers to identify potential sources of error, and by avoiding personal responsibility, it can empower many members of the team to participate in error reduction.

\section{Surgeons' Personal Responsibility for Their Patients' Complications}

Surgeons generally feel a tremendous personal responsibility for complications that occur in surgical patients. Anyone who has been at surgical morbidity and mortality conference knows that the most common question asked is, "What would you have done differently?" This question identifies the surgeon as the agent who potentially could have avoided the complication. Almost three decades ago, sociologist Charles Bosk studied how surgeons deal with errors in their training in his influential book, Forgive and Remember [3]. He observed that surgical treatment links the action of the physician and the response of the patient more intimately than other aspects of medicine. When an internist's patient dies, the natural question colleagues ask is: "What happened?" However, when a surgeon's patient dies, colleagues ask: "What did you do?" [3] This shift reflects the difference in the way surgeons conceptualize their responsibilities to their patients. Although Adedeji and colleagues do not discount the possibility of surgeons' feeling a personal responsibility for complications in their own patients, the account of the ethics of surgical complications provided above does not adequately acknowledge this personal responsibility or the impact this responsibility can have on an individual surgeon.

\section{Burden of Responsibility on the Surgeon}

Most surgeons have heard the saying, "the only surgeon without complications is the surgeon who does not operate." Nevertheless, complications place a heavy burden on surgeons that may not be adequately appreciated by the 
analytical treatment of complications as Adedeji and colleagues have provided. It is not uncommon for a surgeon to stay up late thinking about a difficult upcoming operation. In fact, when most people think about the risks and burdens of surgery, they tend to focus solely on the patient. However, the toll of operating in complex cases where the risk of complications is great should not be underestimated. As Martin Mckneally has astutely pointed out: "The inherent strain of performing operations on patients who are seriously ill takes a toll that is generally underemphasized..." [4]. Acknowledging the burden of complications on individual surgeons is essential to fully understanding surgical complications.

\section{Summary}

The authors of the article in question have provided an important analysis of how ethical principles can be applied to the issues faced by individual surgeons. They have, thereby, provided all surgeons an important challenge to "engage in these issues in greater depth and, in so doing, raise the ethical standards of surgery even higher." Only time will tell whether surgeons do rise to the challenge to improve ethical standards. However, ethical analyses of the important topic of surgical complications will help pave the way toward raising the ethical standards of surgeons and surgery.

\section{References}

1. Simisade A, Sokol DK, Palser T et al (2009) The ethics of surgical complications. World J Surg. doi:10.1007/s00268-008-9907-z

2. Sokol D, Wilson J (2008) What is a surgical complication? World J Surg 32:942-944

3. Bosk C (1979) Forgive and remember: managing medical failure. University of Chicago Press, Chicago, p 30

4. Mckneally M (2009) Surgeons' consent. J Thorac Cardiovasc Surg (forthcoming) 